\title{
Active hospital-based surveillance of invasive pneumococcal disease and clinical pneumonia in infants and young children in two Polish counties
}

Jacek Wysocki ${ }^{1}$, Wojciech Sluzewski², Elane Gutterman ${ }^{3}$, Sylvie Jouve ${ }^{4}$, Michele Moscariello ${ }^{5}$, Ivana Balter ${ }^{4}$

\author{
1Department of Preventive Medicine, Poznan University of Medical Sciences, Poznan, \\ Poland \\ ${ }^{2}$ Department of Infectious Diseases and Pediatric Neurology, Poznan University of \\ Medical Sciences, Poznan, Poland \\ ${ }^{3}$ Via Research, LLC, Princeton Junction, NJ, USA \\ ${ }^{4}$ former employee of Pfizer Inc, Paris, France \\ ${ }^{5}$ Pfizer Inc, Collegeville, PA, USA
}

Submitted: 7 August 2014

Accepted: 21 May 2015

Arch Med Sci 2016; 12, 3: 629-638

DOI: 10.5114 /aoms.2016.59936

Copyright @ 2016 Termedia \& Banach

\section{Abstract}

Introduction: Invasive pneumococcal disease (IPD) incidence, serotype distribution, and antibiotic susceptibility of Streptococcus pneumoniae were estimated in children aged 28 days to $<60$ months.

Material and methods: One-year prospective, hospital-based surveillance was conducted starting on February 15, 2008, at two children's hospitals serving the city and surrounding county of Poznań and Poznański, Poland. Eligible children had fever $\geq 39.0^{\circ} \mathrm{C}$ or physician-suspected IPD. Blood cultures were obtained from all children, cerebrospinal fluid in suspected meningitis cases, and chest radiographs (CXRs) in suspected pneumonia cases. Results: Seven of 1,581 eligible children had confirmed IPD. Estimated IPD incidence per 100,000 children was 11.89 (95\% Cl: 4.78-24.50) overall and 20.1 (95\% Cl: $6.52-46.84)$ in subjects aged 28 days to < 24 months. One S. pneumoniae isolate of each of the following serotypes was obtained: 6B, 14, $23 \mathrm{~A}, 23 \mathrm{~F}$, and 33F. Two isolates were resistant to both trimethoprim-sulfamethoxazole and erythromycin. Clinical pneumonia incidence among children aged 28 days to < 24 months and 24 months to < 60 months was $3,151.3$ (95\% Cl: 2934.7-3379.7) and 962.7 (95\% Cl: 861.2-10,072.9) per 100,000 children, respectively. CXR-confirmed pneumonia rates in the same groups were 1,035.7 (95\% Cl: $913.2-1,170.1)$ and 379.8 (95\% Cl: 317.1-451.3) per 100,000 children, respectively.

Conclusions: IPD is an important cause of morbidity in Poznań and Poznański county, Poland. Among participants aged $<5$ years with fever or suspected IPD, pneumonia was the most common diagnosis and was highest in children aged $<24$ months.

Key words: X-ray-confirmed pneumonia, meningitis, bacteremia, incidence rate.

\section{Introduction}

Streptococcus pneumoniae is a major cause of serious infections, including meningitis, septicemia, pneumonia, and bacteremia. These infec-

\author{
Corresponding author: \\ Jacek Wysocki \\ Department of \\ Preventive Medicine \\ Poznan University \\ of Medical Sciences \\ Smoluchowskiego St 11 \\ 60-179 Poznan, Poland \\ Phone: +48 8612243 \\ Fax: +48 8612242 \\ E-mail: jwysocki@ump.edu.pl
}


tions are associated with high pediatric morbidity and mortality [1]. In children aged $<5$ years, clinical pneumonia is a leading cause of mortality worldwide, accounting for about $19 \%$ of deaths in childhood [2]. Currently, there are 94 pneumococcal serotypes, which are classified according to variations in the bacterial polysaccharide capsule [3]. Differences in invasiveness and disease severity vary by serotype and tend to change over time and across different geographic regions; disease severity is also affected by host-specific characteristics, such as age and underlying medical conditions [4]. Introduction of national immunization programs, such as those implemented in Western Europe, has led to substantial reductions in disease due to $S$. pneumoniae, particularly for serotypes that are covered by pneumococcal conjugate vaccines (PCVs) [5-7].

In 2008, prior to commencing the present study, existing pneumococcal surveillance programs in Central and Eastern Europe were heterogeneous with respect to the populations covered, data sources, types of data collected, and reporting systems. Most studies were based on passive microbiology laboratory surveillance that did not capture all pneumococcal disease, and most used suboptimal standard culture techniques. Therefore, there was a clear need for prospective studies using active surveillance. During the conduct of the present study, the 7-valent pneumococcal conjugate vaccine (PCV7) was not included in the Polish national immunization program free for all children $[8,9]$. Instead, access to the vaccine varied by municipality, and was determined by local authorities and budgets. In 2008, only one locale (Kielce, in the Świętokrzyskie Voivodeship) provided PCV7 as a free-of-charge vaccination for all children born in and after January 2006 [10].

In accordance with a decree from the Polish Ministry of Health, effective October 1, 2008, PCV7 became available for all high-risk children throughout Poland. This decree defines high-risk children as those aged between 2 and 60 months with traumas or central nervous system defects associated with cerebrospinal fluid (CSF) leakage, or those with the following conditions: chronic heart diseases with circulatory insufficiency, immunological-hematological disease, idiopathic thrombocytopenia, acute leukemia, lymphoma, congenital spherocytosis, asplenia, nephritic syndrome (only the genetically determined form), human immunodeficiency virus, or during the period preceding or following a transplantation. Also included are children born prematurely who are aged $<1$ year and have bronchio-pulmonary dysplasia [11]. In Poznań, PCV7 vaccine was also available to children who attend daycare programs (personal communication: J. Wysocki, June 2009). In 2009, a 10-valent pneumococcal conjugate vaccine containing 3 additional serotypes became available in Poland, and in 2011, the 13-valent pneumococcal conjugate vaccine (PCV13) was introduced and replaced PCV7.

Prior to the introduction of a new vaccine, the Active Surveillance of Pneumococcal Epidemiology in Central Eastern Europe (ASPECT) study was designed to estimate the burden of invasive pneumococcal disease (IPD), particularly pneumococcal pneumonia, in children aged 28 days to $<60$ months, in a well-defined surveillance area. This report presents data collected from the only 2 hospitals serving children in Poznań and Poznański county, Poland, from February 15, 2008, to February 14, 2009.

The primary objectives of this study were to estimate the incidence rate of IPD based on the isolation of $S$. pneumoniae from a sterile body site in children aged 28 days to $<60$ months, and to describe the serotype distribution of invasive S. pneumoniae isolates in the surveillance area. The secondary objectives were to estimate the incidence rate of clinical pneumonia and chest radiograph-confirmed pneumonia; estimate case fatality rates for IPD (meningitis, bacteremic pneumonia, septicemia/sepsis, and bacteremia) and clinical and chest radiograph-confirmed pneumonia; describe the antibiotic resistance rates of invasive S. pneumoniae isolates; describe the serotype distribution of resistant S. pneumoniae isolates; assess the neurological sequelae of pneumococcal meningitis; and describe risk factor distribution for IPD.

\section{Material and methods}

This was a prospective, hospital-based, active, targeted surveillance study based on Pneumococcal Vaccines Accelerated Development and Introduction Plan (PneumoADIP) standard case definitions for pneumococcal disease [12]. The surveillance area for this study comprised the city of Poznań and the surrounding Poznański county, which is located in the Wielkopolska Voivodeship, and included two hospitals: the Regional Children's Hospital and the Children's Hospital within the Poznań University of Medical Science. These hospitals are the only hospitals that treat children within the surveillance area. At both hospitals, children must present to the emergency department prior to being admitted. Of note, the Regional Hospital facility has the only designated emergency department for children within the surveillance area. Therefore, parents would not bring their children to the University Hospital unless they received a referral for hospitalization from their primary care physician, or if their child had a chronic condition and was already receiving care from a specialist affiliated with this hospital. 
This study enrolled children aged 28 days to $\leq 36$ months who presented to the hospital with a measured temperature or history of a measured temperature of $\geq 39.0^{\circ} \mathrm{C}$ within $24 \mathrm{~h}$ before screening or clinical suspicion of pneumonia, meningitis, sepsis, bacteremia or other IPD regardless of temperature; or children aged > 36 months to $<60$ months who presented to the healthcare facility with clinical suspicion of pneumonia, meningitis, sepsis, bacteremia, or other IPD regardless of temperature. Informed consent was obtained from all parents or legal guardians of subjects. This study was conducted in accordance with Good Clinical Practices and the ethical principles originating from the Declaration of Helsinki. The protocol, posters, and informed consent form were approved by the ethics committee and/or internal review board at each institution participating in the study. The population of children aged 28 days to $<5$ years residing within Poznań and Poznański county was estimated from government census data as of December 31, 2008 [13].

Quality assurance and quality control mechanisms were set up for periodic monitoring and feedback. Data including the number of enrollees and those eligible but not enrolled (with the reason for non-enrollment noted) were monitored on an ongoing basis to provide feedback on data collection processes.

\section{Data collected}

The following data were collected at the time of study enrollment: demographic information, enrollment diagnosis, history of receipt of pneumococcal vaccine, antibiotic and herbal or traditional medicine use in the previous 7 days, residence, and absence/presence of factors known to be associated with IPD. In addition, one blood specimen was collected upon enrollment, before antibiotic administration if clinically possible, for identification of S. pneumoniae by culture. Cerebrospinal fluid was collected for culture and latex antigen agglutination from all subjects with suspected meningitis. Specimens from other sterile sites were collected as per routine medical practice and cultured using routine methods. All bacteriology samples were cultured at a local laboratory for identification of pathogens according to established standard microbiologic procedures, and each local laboratory reported microbiologic results as per their laboratory's standard operating procedures. Streptococcus pneumoniae isolates were subcultured and sent to a central laboratory for confirmation of identification, serotyping (performed using type-specific sera by Quellung reaction), and antimicrobial susceptibility testing. Antibiotic susceptibility to penicillin, amoxicillin, ampicillin, erythromycin, trimethoprim-sulfame- thoxazole (TMP/Sulfa), ceftriaxone, levofloxacin, and vancomycin was conducted according to standard methodology, including microbroth dilution and/or epsilometer (E-test). Antimicrobial resistance rates were analyzed for all S. pneumoniae isolates tested at the central laboratory and categorized as susceptible, intermediate, or resistant in accordance with the 2007 guidelines from the Clinical and Laboratory Standards Institute [14].

Chest radiographs were obtained for subjects with suspected pneumonia. Results of chest radiographs performed as part of the normal standard of care for subjects with pneumonia clinical syndrome, taken within $72 \mathrm{~h}$ of study enrollment, could be used. For abnormal results, the presence of lobar consolidation, pleural effusion, both, or "other" was documented. Radiologists used the World Health Organization (WHO) guidelines to evaluate chest radiographs [15]. In those with pneumonia clinical syndrome, C-reactive protein (CRP) was tested at the local laboratory with a threshold of $\geq 40 \mathrm{mg} / \mathrm{l}$ [16]. Additional information on diagnosis and vital status was collected for all hospitalized subjects, all subjects whose culture(s) yielded S. pneumoniae, and/or all subjects whose inclusion criteria included clinical suspicion of meningitis. This information was collected on day 10 or on the day of hospital discharge, whichever occurred first. For subjects with a diagnosis of probable or definite pneumococcal meningitis, a neurological examination was conducted between 6 and 9 months after enrollment for evaluation of neurological sequelae.

\section{Diagnoses}

Invasive pneumococcal disease was defined as the identification of S. pneumoniae from a sterile body fluid (e.g. blood, CSF, pleural fluid, peritoneal fluid, joint fluid, pericardial fluid, lung, bone, or bone marrow) by culture: the isolate was confirmed as S. pneumoniae by the central laboratory if the isolate was sent to the central laboratory; otherwise local identification served as confirmation. Cases with a positive culture could have multiple culture sources (during the same episode), or even two (positive) blood cultures. Subjects were diagnosed with pneumococcal meningitis if the CSF sample was positive for S. pneumoniae antigen by latex agglutination antigen detection test, or had a positive Gram stain for cocci with all other biochemical CSF criteria for abnormal CSF.

\section{Incidence rate of invasive pneumococcal disease}

Invasive pneumococcal disease incidence rates were computed per 100,000 children in the at-risk population, with $95 \%$ confidence intervals (Cls) 
based on the Poisson distribution. Age- and gender-specific IPD incidence rates were computed. Separate incidence rates were computed for each diagnosis within the IPD hierarchy. These incidence rates were also shown by diagnosis, and by age and gender. Hospital-based case fatality rates for IPD were calculated as the number of deaths (within each condition) divided by all the children with the respective conditions.

\section{Serotype distribution of Streptococcus pneumoniae isolates}

Frequency distributions that provided the number and percentage of IPD cases per serotype were given. Breakdowns of serotype by diagnosis were determined. Frequency distributions were provided for all S. pneumoniae serotypes identified. Isolates were also grouped and categorized by serotypes included in PCV7, 10-valent pneumococcal conjugate vaccine (PCV10), and PCV13.

\section{Statistical analysis}

Statistical analyses were performed using SAS software (version 9.1; SAS Institute, Cary, NC, USA). Analyses of incidence addressed all cases captured during a single year of surveillance from

Table I. Patient demographics

\begin{tabular}{|c|c|c|}
\hline $\begin{array}{l}\text { Demographic } \\
\text { variable }\end{array}$ & $\begin{array}{c}\text { Study } \\
\text { population } \\
(N=1581)\end{array}$ & $\begin{array}{l}\text { IPD cases } \\
(N=7)\end{array}$ \\
\hline \multicolumn{3}{|l|}{ Age [months]: } \\
\hline Mean \pm SD & $17.8 \pm 14.5$ & $18.9 \pm 10$ \\
\hline Median & 14.01 & 20 \\
\hline Range & $1-59$ & $6-35$ \\
\hline \multicolumn{3}{|l|}{ Gender, $n(\%)$ : } \\
\hline Female & $666(42.1)$ & $4(57.1)$ \\
\hline Male & 915 (57.9) & $3(42.9)$ \\
\hline \multicolumn{3}{|c|}{ Gestational age at birth [weeks]: } \\
\hline$n$ & 1548 & 7 \\
\hline Mean \pm SD & $38.7 \pm 2.2$ & $39.0 \pm 1.0$ \\
\hline Median & 39.0 & 39.0 \\
\hline Range & $26-44$ & $38-40$ \\
\hline \multicolumn{3}{|c|}{ Birth weight [g]: } \\
\hline$n$ & 1570 & 7 \\
\hline Mean \pm SD & $3342.9 \pm 606.7$ & $3621.4 \pm 625.6$ \\
\hline Median & 3380.0 & 3700.0 \\
\hline Range & $740-5000$ & 2480-4320 \\
\hline
\end{tabular}

February 15, 2008, to February 14, 2009. Individuals could be enrolled in the study more than once provided that the presenting illnesses reflected independent episodes and all eligibility criteria were met. Therefore, each enrollment was considered as a separate subject/episode and analyzed accordingly. Unless otherwise noted, the episode was the unit of analysis.

The estimated number of children in the atrisk population (i.e., those aged 28 days to $<60$ months within Poznań and Poznański county) served as the denominator for IPD and other incidence rates computed in this study. Numbers for children $\geq 1$ year of age were taken from government census data as of December 31, 2008, while population data for children aged $<1$ year were adjusted to exclude the estimated number of children aged $<28$ days. Accordingly, the total atrisk population was estimated as 58,877 children. Due to the small number of IPD-positive subjects, statistical comparisons between the study population and subset of children with IPD were not feasible.

\section{Results}

There were 1581 subjects evaluated in the study population, and 7 subjects with IPD (Table I). Overall, 1576 (99.7\%) subjects completed the study. Five subjects did not complete the study: 1 because of death, 1 withdrew consent, and 3 had protocol violations. A total of 1516 (95.9\%) subjects were hospitalized and all 7 subjects with IPD were hospitalized. The mean age was 17.8 months for the study population and 18.9 months for subjects with IPD. In the study population there were slightly more males than females, whereas among the IPD cases there were 4 females and 3 males.

Diagnoses for the study population are listed in Table II. The most common diagnosis was pneumonia clinical syndrome (69.7\%). Of the $3 \mathrm{sub}$ jects with meningitis clinical syndrome, 2 (66.7\%) returned for neurological evaluation, with neurological sequelae of seizure observed in 1 subject.

\section{Invasive pneumococcal disease cases}

Among the 7 subjects with IPD, 1 had confirmed pneumococcal meningitis and sepsis; 1 had definite pneumococcal meningitis and bacteremia; 1 had sepsis and pneumonia clinical syndrome; 1 had sepsis, pneumonia clinical syndrome, and otitis catarrhalis; 1 had pneumonia clinical syndrome and bacteremia; 1 had pneumonia clinical syndrome, bacteremia, sinusitis, and anemia; and 1 had pneumonia clinical syndrome, purulent otitis media, and acute gastroenterocolitis. As shown in Table III, the hierarchical diagnoses in IPD subjects were pneumonia clinical syndrome (42.9\%), 
Table II. Subject diagnoses ${ }^{a}$

\begin{tabular}{|lc|}
\hline Variable & $N(\%)$ \\
\hline Total & $1581(100)$ \\
\hline Total eligible for diagnosis & $1525(96.5)$ \\
\hline Nonmissing diagnosis ${ }^{b}$ & $1522(96.3)$ \\
\hline Meningitis clinical syndromec: & $3(0.2)$ \\
\hline Definite pneumococcal meningitis & $2(0.1)$ \\
\hline Probable pneumococcal meningitis & $1(0.1)$ \\
\hline Sepsis & $8(0.5)$ \\
\hline Pneumonia clinical syndrome & $1061(69.7)$ \\
\hline Osteomyelitis & $29(1.9)$ \\
\hline Bacteremia & $1(0.1)$ \\
\hline Cellulitis & $995(65.4)$ \\
\hline Other & (1) \\
\hline
\end{tabular}

anly subjects who were eligible for a diagnosis were counted. A subject was eligible for a diagnosis if they were hospitalized, had a positive culture for S. pneumoniae, or had an initial diagnosis of meningitis clinical syndrome. A subject could be included in more than one diagnosis. ${ }^{b}$ Nonmissing diagnosis is the unit of analysis; the denominator is nonmissing diagnosis value. 'Meningitis clinical syndrome subsets were definite meningitis, nonpneumococcal, and other meningitis.

meningitis clinical syndrome (28.6\%), and sepsis (28.6\%).

\section{Previous pneumococcal vaccine}

In the study population, 156 of 1581 subjects were vaccinated with a PCV with a mean of 1.9 \pm 0.9 doses, and a mean age at first vaccination of $11.7 \pm 12.1$ months; 5 subjects were vaccinated with pneumococcal polysaccharide vaccine (PPV). In the IPD cases, 1 of 7 subjects was vaccinated with 3 doses of PCV7 (administered at 1, 3, and 4 months) and developed IPD caused by serotype $33 F$, which is not included in PCV7. No subject with IPD was vaccinated with PPV.

\section{Prior antibiotic treatment and presence of risk factors for invasive pneumococcal disease}

Antibiotics were used during the previous 7 days in $42.7 \%$ of the study population and in $57.1 \%$ of subjects with IPD. Overall, $81 \%$ of the study population and $85.7 \%$ of the IPD patients resided in an urban area.

In the study population, 314 (20\%) children attended daycare or kindergarten within the past 3 months, 643 (41.2\%) lived in a household with a smoker, and 544 (34.8\%) were breast fed for $<2$ months; the mean number of people in the household was 4.2 (range: 1-13), the mean number of children aged $<60$ months in the household was
Table III. Clinical and bacteriological description of IPD cases $(n=7)$

\begin{tabular}{|c|c|}
\hline Variable & $N(\%)$ \\
\hline Total eligible for diagnosis ${ }^{a}$ & $7(100)$ \\
\hline \multicolumn{2}{|l|}{ Hierarchical diagnosis ${ }^{\mathrm{b}}$ : } \\
\hline Meningitis clinical syndrome & $2(28.6)$ \\
\hline Sepsis & $2(28.6)$ \\
\hline Pneumonia clinical syndrome & $3(42.9)$ \\
\hline \multicolumn{2}{|l|}{ Serotypes: } \\
\hline $6 \mathrm{~B}$ & $1(14.3)$ \\
\hline 14 & $1(14.3)$ \\
\hline $23 A^{c}$ & $1(14.3)$ \\
\hline $23 \mathrm{~F}$ & $1(14.3)$ \\
\hline $33 \mathrm{~F}^{\mathrm{c}}$ & $1(14.3)$ \\
\hline Missing & $2(28.6)$ \\
\hline \multicolumn{2}{|l|}{ Antibiotic resistance ${ }^{\mathrm{d}, \mathrm{e}}$ : } \\
\hline Erythromycin $(n=5)^{f}$ & $2(40.0)$ \\
\hline Trimethoprim-sulfamethoxazole $(n=5)^{f}$ & $2(40.0)$ \\
\hline
\end{tabular}

${ }^{a}$ Only subjects who were eligible for a diagnosis were counted. A subject was eligible for a diagnosis if they were hospitalized, had a positive culture for S. pneumoniae, or had an initial diagnosis of meningitis clinical syndrome. ${ }^{b} A$ subject was only included in one hierarchical diagnosis. Hierarchical diagnosis assigned a single diagnosis to each subject that represented the highest or most severe infection within the hierarchy. "Serotypes not included in pneumococcal conjugate vaccines. "For subjects with multiple cultures, the most resistant result was counted. ${ }^{e} T$ Two subjects had at least one culture that did not have serotype results. fPercentages are based on the denominator of each row. The denominator is nonmissing susceptibility values for each antimicrobial. IPD invasive pneumococcal disease.

1.4 (range: $1-4$ ), and the mean number of sleeping rooms in the household was 2.3. Among IPD cases, $3(42.9 \%)$ children attended daycare or kindergarten within the past 3 months, 2 (28.6\%) lived in a household with a smoker, and 3 (42.9\%) were breast fed for $<2$ months. The mean number of people in the household was 3.6 (range: 3-5), the mean number of children aged $<60$ months in the household was 1.4 (range: $1-2$ ), and the mean number of sleeping rooms in the household was 2.2. Whereas $10 \%$ of children in the study population were born prematurely ( $<37$ weeks), none of the children with IPD were born prematurely.

\section{Invasive pneumococcal disease incidence rate}

The overall IPD incidence rate was 11.89 (95\% Cl: 4.78-24.50) per 100,000 children. The IPD incidence rates per 100,000 children were 20.07 (95\% Cl: 6.52-46.84) for subjects aged 28 days to < 24 months vs. 5.89 (95\% Cl: 0.71-21.27) for 
those aged 24 months to $<60$ months. When categorized by age groups, the IPD incidence rates per 100,000 children were $29.65(95 \% \mathrm{Cl}$ : 3.59-107.10) for those aged 6 months to < 12 months; 24.17 (95\% Cl: 4.98-70.62) for subjects aged 12 months to < 24 months; and 16.79 (95\% Cl: 2.03-60.65) for those aged 24 months to $<36$ months. No cases of IPD were observed in the 28 days to $<6$ months or 36 months to $<60$ months age groups. In addition, the IPD incidence rates per 100,000 children were 9.89 (95\% Cl: 2.04-28.90) for males and 14.02 (95\% Cl: 3.82-35.89) for females. Incidence rates of IPD per 100,000 children by hierarchy of diagnosis were 5.10 (95\% Cl: 1.05-14.89) for pneumonia clinical syndrome and 3.40 (95\% Cl: 0.41-12.27) for both pneumococcal meningitis and sepsis.

\section{Bacteriology and antibiotic resistance}

Almost all study subjects (99.6\%) had cultures performed, and these were positive in $23.3 \%$ of samples. Overall, $2.4 \%$ (9 of 370) of all organisms were identified as S. pneumoniae. The other most frequently isolated organisms (Staphylococcus epidermidis (20.5\%), Micrococcus species (12.4\%), Micrococcus luteus (10.8\%), Staphylococcus hominis (7.3\%), and Staphylococcus haemolyticus (5.9\%)) were presumably contaminants.

Among the 7 subjects with IPD, there were 7 S. pneumoniae isolates from the blood and 1 from the CSF. There was also one additional culture that was positive for S. pneumoniae; however, this individual failed to meet the eligibility criteria and was not included in the analysis. Serotypes were determined for 5 of the isolates (Table III).

Serotype 23A was isolated from 1 subject with a diagnosis of sepsis, pneumonia clinical syndrome, and otitis catarrhalis; and serotype 33F was isolated from 1 subject with a diagnosis of definite pneumococcal meningitis and bacteremia. Serotype 14 was isolated from 1 subject with a diagnosis of definite pneumococcal meningitis, and serotype $6 \mathrm{~B}$ was isolated from 1 subject with pneumonia clinical syndrome. Serotype $23 \mathrm{~F}$ was isolated from 1 subject with pneumonia clinical syndrome. Serotypes were not determined in $1 \mathrm{sub}$ ject with sepsis and 1 subject with pneumonia clinical syndrome.

The percentage of $S$. pneumoniae serotypes included in 7-valent (4, 6B, 9V, 14, 18C, 19F, 23F), 10 -valent $(1,4,5,6 \mathrm{~B}, 7 \mathrm{~F}, 9 \mathrm{~V}, 14,18 \mathrm{C}, 19 \mathrm{~F}, 23 \mathrm{~F})$, and 13-valent $(1,3,4,5,6 \mathrm{~A}, 6 \mathrm{~B}, 7 \mathrm{~F}, 9 \mathrm{~V}, 14,18 \mathrm{C}, 19 \mathrm{~A}$, $19 F, 23 F)$ PCV was $60 \%$ for each vaccine because only serotypes $6 \mathrm{~B}, 14$, and $23 \mathrm{~F}$ were identified.

Antimicrobial resistance rates were $40.0 \%$ (2 of 5 samples) for both TMP/Sulfa and erythromycin (Table III). No decreased susceptibility was observed for penicillin, amoxicillin, ampicillin, ceftriaxone, levofloxacin, or vancomycin. Isolates of serotypes $6 \mathrm{~B}$ and 14 were resistant to erythromycin, and isolates of serotypes $6 \mathrm{~B}$ and $23 \mathrm{~F}$ were resistant to TMP/Sulfa.

\section{Incidence of pneumonia}

When categorized by different age groups, in cidence rates for clinical, chest radiograph-confirmed, and CRP chest radiograph-confirmed pneumonia in the study population were highest for the youngest age groups ( 28 days to $<24$ months) and, in general, decreased with increasing age (Table IV). Among all study subjects and subjects with IPD, $98 \%$ and 100\%, respectively, had chest radiographs performed to rule out pneumonia. Of the 1112 subjects with clinical pneumonia, 387 were diagnosed with chest radiograph-confirmed pneumonia. During the surveillance period, only $24.5 \%$ (387 of 1581) of subjects were diagnosed with chest radiograph-confirmed pneumonia (Table IV).

\section{Case fatality rates}

In the study population, case fatality rates were $33.3 \%$ (1 of 3 subjects) among those with a diagnosis of meningitis, $12.5 \%$ (1 of 8 subjects) for those with a diagnosis of sepsis, and $0.1 \%$ ( 1 of 1112 subjects) for those with a diagnosis of clinical pneumonia. No deaths were attributable to IPD.

\section{Safety}

No protocol-related adverse events or serious adverse events were reported, and there were no withdrawals from the study due to a protocol-related adverse event. There were no protocol-related deaths.

\section{Discussion}

This study assessed the incidence rate of IPD over a 1-year period in children aged 28 days to $<60$ months in the city of Poznań and Poznański county, Poland. Seven cases of IPD were identified in the 1581 enrolled subjects, yielding an overall IPD incidence rate per 100,000 children in this study of 11.89 , which was lower than the incidence rate of 17.60 in children aged 0-59 months reported by Grzesiowski et al. [17] in a study conducted in 5 districts in Poland. The latter incidence rate was based on the identification of a total of 134 IPD cases among 88,487 children enrolled in the study between January 2003 and December 2004. Importantly, whereas the Grzesiowski et al. study included both culture-positive cases $(n=26)$ and culture-negative samples confirmed by polymerase chain reaction (PCR; $n=108$ ), the current study calculated IPD incidence rates 


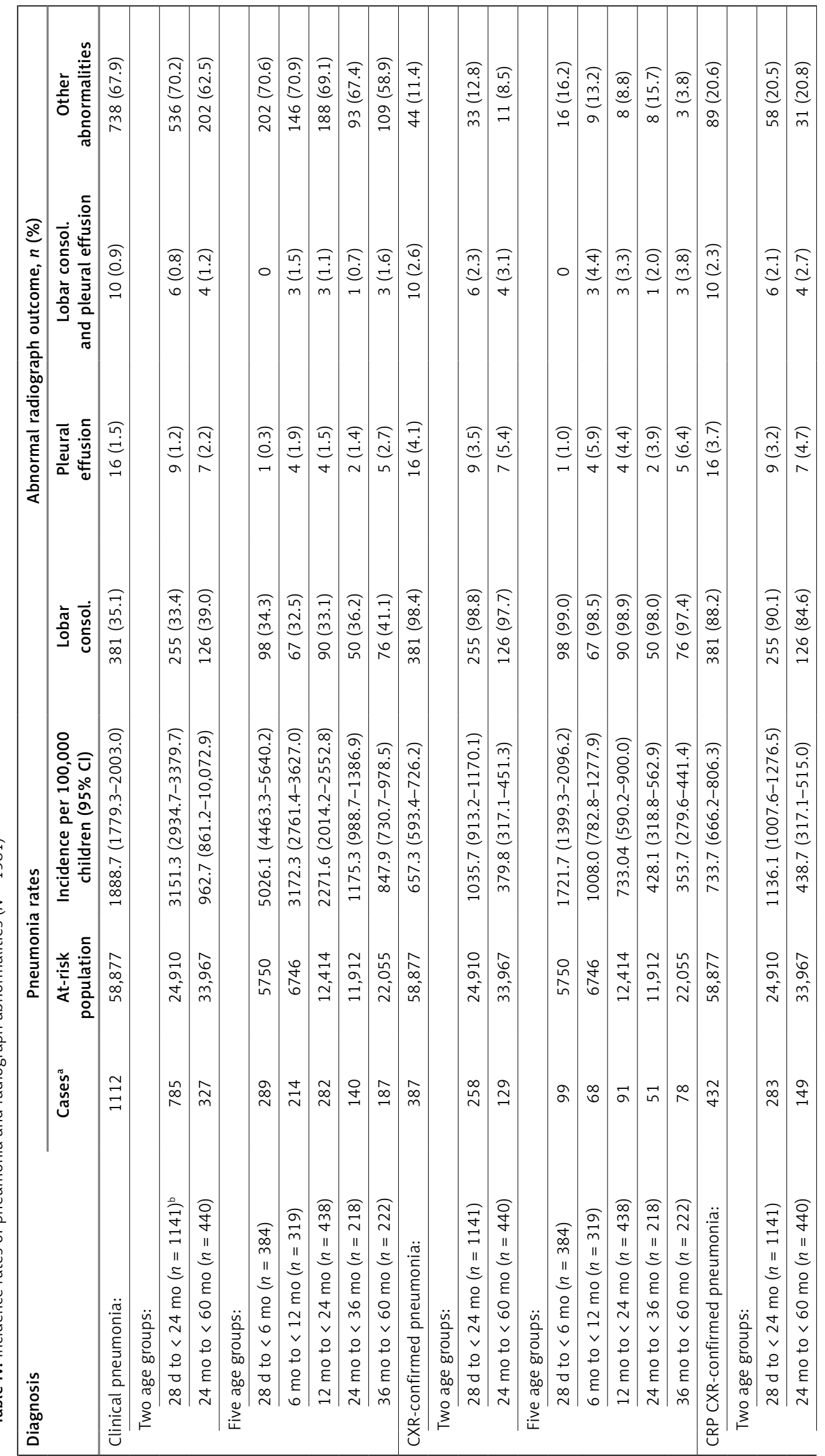




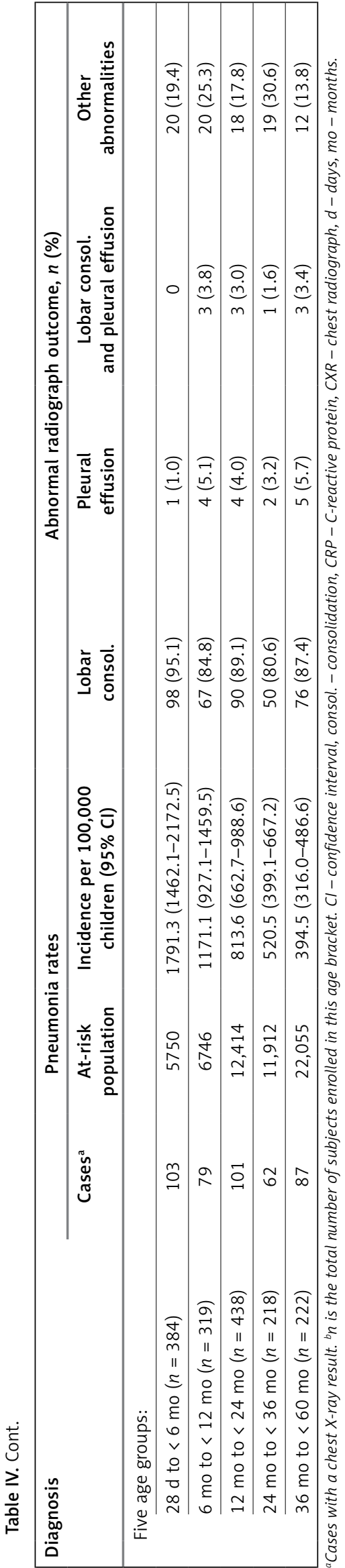

for culture-positive cases only $(n=7)$. Although re-analysis based on PCR results was planned for this study, it was not performed due to sample stability issues, and this was one of the limitations of this study. A study by Skoczyńska et al. [18] conducted throughout Poland between 2006 and 2009 reported much lower incidence rates. In this study, the incidence rates per 100,000 children aged 0-4 years increased from 0.9 in 2006 to 2.85 in 2009. The lower IPD incidence rates observed in the Skoczyńska et al. study may reflect the fact that culture practices were linked to standard of care in Poland. Although CSF is commonly cultured in cases of suspected meningitis, blood is infrequently cultured in Poland. Therefore, when IPD incidence rates are calculated based on cultures performed based on standard of care in Poland, overall IPD incidence rates can be expected to be low and meningitis is likely to account for a substantial portion of IPD cases. Although PCV7 was registered in Poland in 2001, it was unavailable until 2005. Use of PCV7 for high-risk children starting in October 2008 may have decreased the incidence of IPD. In this study, 9.9\% of enrolled children received at least one dose of PCV7.

Three of five of the S. pneumoniae serotypes found in this study (serotypes 6B, 14, and 23F) were also frequently reported in other published studies of IPD in children aged $<5$ years in Poland $[12,13]$. Of the serotypes isolated in this study, $60 \%$ are included in PCV7, PCV10, and PCV13. The percentage of $S$. pneumoniae serotypes included in PCV7 in this study (60.0\%) is comparable to those previously reported $(68.7-77.3 \%)[17,18]$. However, $40 \%$ (2 of 5) of isolates were serotypes (23A and 33F) not covered by the PCV vaccines that are currently available.

Antimicrobial resistance of $S$. pneumoniae was observed for TMP/Sulfa and erythromycin. Erythromycin resistance $(40.0 \%)$ in the current study was comparable to that seen in another study in Poland in children aged < 5 years (31\%) [17]. None of the isolates was resistant to penicillin or cephalosporin.

The annual incidence rate of chest radiograph-confirmed pneumonia in the current study $(657.30$ per 100,000$)$ is lower than the rate reported in the Kielce area prior to and following comprehensive vaccination with PCV7. Patrzatek et al. [10] reported 331 chest radiograph-confirmed pneumonia cases in 16,377 children aged $<5$ years (annual incidence rate: 2021.13 per 100,000 ) in 2004-2005 in Kielce, Poland, prior to implementation of the PCV7 vaccination program. In the 2 years following implementation of a compulsory, free-of-charge PCV7 vaccination program in Kielce, only 146 cases were identified in a population of 17,105 (annual incidence rate: 853.55 per 100,000). 
Use of a CRP chest radiograph increased the detection of pneumonia by $10 \%$, from 657.3 to 733.7 per 100,000 . A positive CRP chest radiograph was defined as chest radiograph-confirmed pneumonia or clinical pneumonia with an abnormal ("other") chest radiograph result plus CRP $\geq 40 \mathrm{mg} / \mathrm{dl}$. Although this assay appears to increase diagnostic sensitivity, opinions concerning the use of acute phase reactants to distinguish bacterial versus viral etiology vary in the literature [16]. A recent meta-analysis that included 1230 children with pneumonia indicated that CRP serum concentrations $>40-60 \mathrm{mg} / \mathrm{l}$ were associated with bacterial pneumonia [19]. Some data suggest that determining CRP levels ( $\geq 40 \mathrm{mg} / \mathrm{dl}$ ) could reduce the risk of underestimating the burden of pneumococcal pneumonia [20].

There are several issues which may limit the results of this study. Although data were available from 1 complete year, longer surveillance would have minimized the effect of year-to-year fluctuations. Also, multiple factors may have contributed to an underestimation of the actual incidence of IPD in the designated surveillance area. Based on the study protocol, cases of IPD and pneumonia were limited to patients who presented to the hospital and did not include patients treated at outpatient health care facilities. Although the most seriously ill patients were likely to present to the 2 participating hospitals, by design, this study excluded patients treated by physicians in primary care clinics and those never referred for hospital services. In Poland, because many children are treated with antibiotics before being referred to a hospital, this likely reduced the chance to obtain a positive blood culture. Furthermore, at both the Regional Hospital and University Hospital, it was not feasible to perform all or most of the enrollment procedures in the emergency department as planned. There was an official hospital policy that prohibited taking blood samples (other than finger sticks) in the emergency department, which necessitated movement of patients to inpatient services for this procedure. This minimized the ability to enroll hospitalized children before they were transferred to separate inpatient services, and led to low enrollment of eligible subjects in the study. Moreover, parents of children who were not hospitalized were reluctant to bring their children to inpatient services. In addition, enrollment may have been limited by long waiting times and lack of a private enrollment space. At the Regional Hospital, the proportion enrolled among eligible, hospitalized children was about $75 \%$. In contrast, $<5 \%$ of eligible nonhospitalized children were enrolled at this facility. The enrollment proportion among eligible hospitalized patients at the University Hospital was just under $60 \%$. Therefore, it is likely that hospital-based incidence within the surveillance area was underestimated by suboptimal enrollment.

Although WHO guidelines [21] were provided to radiologists interpreting chest radiographs, a certain degree of interindividual and intraindividual variability in chest radiograph interpretation, and the fact that only lobar consolidation and pleural effusion were acceptable as chest radiograph-confirmed cases, might have led to underestimation of the number of cases. Chest radiographs interpreted as "abnormal" were not collected or re-analyzed; therefore, the number of pneumonia cases might have been underestimated or overestimated.

Overall, $99.6 \%$ of subjects had a culture performed. Of these, only 7 subjects had cultures positive for S. pneumoniae, with the other isolates considered primarily contaminants. The volume of blood drawn, vein accessibility, dilution, number of cultures taken, delays in processing cultures, blood culture technique (including skin preparation, choice of culture site, timing, and choice of blood culture bottle), and system are all factors that can influence the ability to obtain pathogens from blood cultures. Prior antibiotic use by $42.7 \%$ of subjects in the study may also have hampered the ability to isolate $S$. pneumoniae. Given the small number of cases in this study, details on serotype distribution are inconclusive. In addition, the calculated incidence is likely to be an underestimate due to the insufficient enrollment of eligible patients and the limited sensitivity of culture methods for identification. A final limitation is that because the surveillance population comprised two contiguous counties, the city of Poznań and the surrounding Poznański county, the results reported here are not generalizable to Poland overall or other areas within Poland.

In conclusion, this hospital surveillance study identified pneumonia as a common diagnosis in children aged $\leq 5$ years presenting to hospitals in Poznań, Poland, and the incidence of pneumonia was highest in the youngest children. Rates of IPD were comparable with other studies conducted in Poland. As the surveillance area comprised only 2 counties, the results reported here should not be generalized to Poland overall. Despite limitations, this study underlines the importance of improved, continuing, active, prospective surveillance of the burden of pneumococcal disease, serotype distribution, and antibiotic resistance in Poland.

\section{Acknowledgments}

Medical writing support was provided by Nancy Price, PhD, at Excerpta Medica and Susan E. DeRocco, PhD, at Complete Healthcare Communications, LLC, and was funded by Pfizer Inc. Local microbiology examinations were performed 
by Pawel Gruszczynski, PhD, of the Wielkopolska Center for Pulmonology and Thoracic Surgery of Eugenia and Janusz Zeyland, Poznań, Poland.

This study was sponsored by Wyeth, which was acquired by Pfizer Inc in October 2009.

\section{Conflict of interest}

JW received travel grants for international scientific conferences and honoraria for lectures from Wyeth and Pfizer. WS has no conflicts to disclose. EG is the President of Via Research, LLC, which was contracted by Pfizer Inc to provide epidemiologic support and surveillance assessments for the study. SJ and IB were employees of Pfizer Inc at the time of the study. MM is an employee of Pfizer.

\section{References}

1. World Health Organization. Pneumococcal conjugate vaccine for childhood immunization - WHO position paper. Wkly Epidemiol Rec 2007; 82: 93-104.

2. Rudan I, Boschi-Pinto C, Biloglav Z, Mulholland K, Campbell $\mathrm{H}$. Epidemiology and etiology of childhood pneumonia. Bull World Health Organ 2008; 86: 408-16.

3. Song JY, Nahm MH, Moseley MA. Clinical implications of pneumococcal serotypes: invasive disease potential, clinical presentations, and antibiotic resistance. J Korean Med Sci 2013; 28: 4-15.

4. Berg S, Trollfors B, Persson E, et al. Serotypes of Streptococcus pneumoniae isolated from blood and cerebrospinal fluid related to vaccine serotypes and to clinical characteristics. Scand J Infect Dis 2006; 38: 427-32.

5. Munson S, Lambrelli D, Raluy D, et al. The burden and reportable causative agents associated with hospitalized pneumonia, meningitis, and septicemia in Norway (2008-2009). Pneumonia 2014; 3: 172.

6. Rodenburg GD, de Greeff SC, Jansen AG, et al. Effects of pneumococcal conjugate vaccine 2 years after its introduction, the Netherlands. Emerg Infect Dis 2010; 16: 816-23.

7. van Deursen AM, van Mens SP, Sanders EA, et al. Invasive pneumococcal disease and 7-valent pneumococcal conjugate vaccine, the Netherlands. Emerg Infect Dis 2012; 18: 1729-37.

8. European Centre for Disease Prevention and Control. Annual epidemiological report on communicable diseases in Europe - 2009. Available at: http://www.ecdc. europa.eu/en/publications/Publications/Forms/ECDC DispForm.aspx?ID=452. Accessed June 19, 2013.

9. De Carvalho Gomes H, Muscat M, Monnet DL, Giesecke J, Lopalco PL. Use of seven-valent pneumococcal conjugate vaccine (PCV7) in Europe, 2001-2007. Euro Surveill 2009; 14: $\mathrm{pii}=19159$.

10. Patrzałek M, Albrecht P, Sobczynski M. Significant decline in pneumonia admission rate after the introduction of routine $2+1$ dose schedule heptavalent pneumococcal conjugate vaccine (PCV7) in children under 5 years of age in Kielce, Poland. Eur J Clin Microbiol Infect Dis 2010; 29: 787-92.

11. Case definitions for pneumococcal syndromes and other severe bacterial infections. Clin Infect Dis 2009; 48 Suppl 2: S197-202.
12. PneumoADIP Core Definitions for Pneumococcal Syndromes. Mombasa, Kenya: 2003; updated 2006.

13. Central Statistical Office of Poland-GUS Available at: http://www.stat.gov.pl/gus/index_ENG_HTML.htm. Accessed June 19, 2013.

14. Clinical and Laboratory Standards Institute. CLSI publishes revised standard on methods for antimicrobial susceptibility testing of anaerobic bacteria (M11-A8). Available at: http://www.clsi.org/clsi-publishes-revisedstandard-on-methods-for-antimicrobial-susceptibility-testing-of-anaerobic-bacteria-m11-a8/. Accessed June 19, 2013.

15. World Health Organization Pneumonia Vaccine Trial Investigators' Group. Standardization of interpretation of chest radiographs for the diagnosis of pneumonia in children. Geneva 2001.

16. British Thoracic Society Standards of Care Committee. British Thoracic Society guidelines for the management of community acquired pneumonia in childhood. Thorax 2002; 57 Suppl 1: i1-24.

17. Grzesiowski P, Skoczynska A, Albrecht P, et al. Invasive pneumococcal disease in children up to 5 years of age in Poland. Eur J Clin Microbiol Infect Dis 2008; 27: 883-5.

18. Skoczyńska A, Sadowy E, Bojarska K, et al. The current status of invasive pneumococcal disease in Poland. Vaccine 2011; 29: 2199-205.

19. Flood RG, Badik J, Aronoff SC. The utility of serum C-reactive protein in differentiating bacterial from nonbacterial pneumonia in children: a meta-analysis of 1230 children. Pediatr Infect Dis J 2008; 27: 95-9.

20. Madhi SA, Kohler M, Kuwanda L, Cutland C, Klugman KP. Usefulness of C-reactive protein to define pneumococcal conjugate vaccine efficacy in the prevention of pneumonia. Pediatr Infect Dis J 2006; 25: 30-6.

21. World Health Organization. Standardization of interpretation of chest radiographs for the diagnosis of pneumonia in children. World Health Organization Pneumonia Vaccine Trial Investigators' Group. Available at: http://whqlibdoc.who.int/hq/2001/WHO_V\&B_01.35. pdf. Accessed June 26, 2013. 